\title{
Color on Demand - Color-Tunable OLEDs for Lighting and Displays
}

\author{
Markus Fröbel ${ }^{1}$, Tobias Schwab ${ }^{1}$, Mona Kliem ${ }^{1}$, Simone Lenk ${ }^{1}$, \\ Karl Leo ${ }^{1}$, Sebastian Reineke ${ }^{1}$, Malte C. Gather ${ }^{1,2}$
}

${ }^{1}$ Dresden Integrated Center for Applied Physics and Photonic Materials and Institute of Applied Physics, Technische Universität Dresden, Dresden, Germany

${ }^{2}$ Organic Semiconductor Centre, SUPA, School of Physics and Astronomy, University of St Andrews, St Andrews, U.K.

\begin{abstract}
A device concept for highly efficient OLEDs is introduced that allows to tune the emission color of the device over a broad range of the CIE color gamut. The approach exploits the different polarities of the positive and negative half-cycles of an alternating current driving signal to independently address two vertically stacked emission units with complementary color. Ultrathin metal electrodes fabricated by a wetting layer approach are used to achieve good electrical contact to each stack with minimal impact on optical performance.
\end{abstract}

Author Keywords

OLED; color tuning; white; stacked; ultrathin metal electrode; alternating current driving; lighting; display 


\section{Introduction}

In recent years, organic light-emitting diodes (OLEDs) have evolved into a mature technology and are now widely used in various display applications, such as mobile phones, television, and wearable devices. OLEDs provide an internal charge-to-photon conversion efficiency of nearly $100 \%$ and deliver homogeneous emission over large areas, making them also promising candidates for new lighting applications [1]. White OLEDs, in particular, offer great potential for energy-efficient general illumination and luminous efficacies of more than 90 $\mathrm{lm} / \mathrm{W}$, comparable to the best fluorescent tubes, have already been reported [2-4]. The spectrally broad emission of organic emitter molecules is advantageous in white OLEDs, as it leads to a high color rendering index and produces white light that is perceived as visually appealing. Furthermore, OLED-based light sources can be made mechanically flexible and transparent, offering new opportunities for architecture, visual art and decoration. Finally, because the emission is over an extended area, OLED light is experienced as a pleasantly glare-free light whereas inorganic LED based luminaires show a more directed emission that have a tendency to produce glare.

Conventional OLEDs are driven with a direct current (DC) signal. The electricity grid, however, provides alternating current (AC) power with a frequency of $50 / 60 \mathrm{~Hz}$ which means an AC-to-DC power converter is necessary in order to power a DC OLED directly from the main grid. Driving schemes using a time-varying signal are, on the other hand, highly attractive, offering more possibilities to interact with a device. By applying the appropriate circuitry, the emission color and intensity of a light source can be controlled during device operation, for example, to switch between cold-white illumination for productive work environments and warm-white light to create a more relaxing atmosphere. Reliable real-time tunability of the emission color would impart further momentum to OLED technology on its way to becoming a widespread source of general illumination.

Thus far, reports on AC driven OLEDs demonstrate relatively modest efficiencies, especially for capacitively coupled AC-OLEDs, operating in a fully insulated mode, i.e. without charge injection from external electrodes [5-8]. For this capacitively coupled configuration, the most promising approach in terms of brightness and efficiency is a p-i-n based architecture in which a single emissive unit is surrounded by doped hole and electron transport layers and by a pair of insulating layers; the whole stack is then sandwiched between two electrodes [9-14]. While in a truly capacitively coupled structure, no charges are injected from the electrodes, the presence of charge within the device is still necessary to form excitons. In AC OLEDs using a p-i-n architecture these charges are provided by the doped transport layers, which serve as a reservoir for electrons (n-doped electron transport layer) and holes (p-doped hole transport layer), as well as by surface states at the interface between insulating layers and transport layers.

We recently reported on an alternative AC driven OLED structure (referred to as AC/DC OLED) that comprises two p-i-n OLEDs stacked on top of each other that each have direct injection electrodes which are connected in a special manner as schematically illustrated in Fig. 1 [15]. This configuration is different from stacked multi-color OLEDs reported earlier [16], in that the special contact configuration allows addressing each OLED independently with a two-wire (rather than a three-wire) external connection (see below). In addition, we use an ultrathin metal electrode produced via a wetting layer approach to form the transparent intermediate contact (E2 in Fig. 1). The wetting layer yields ultrathin metal electrodes that achieve a very favorable combination of conductivity and optical performance [17-19].

\section{AC/DC OLED Layout}

For the results discussed here, we employed an AC/DC OLED device with an architecture that comprises a yellow phosphorescent pinOLED stacked on top of a blue fluorescent pin-OLED. Internally, our devices have three electrodes (labeled E1, E2, and E3 in Fig. 1), however, E1 and E3 are electrically connected inside the device so that they are at the same potential upon application of a voltage and can be addressed via a single external contact. E2 is the independent counter-electrode.

The polarity between E1/E3 and E2 controls if the blue or the yellow emitting sub-unit of the device is active: When putting E1/E3 on positive potential with respect to E2, we observe blue emission because the blue sub-unit is biased in forward direction while the yellow sub-unit is biased in reverse direction and thus shows negligible current density and no yellow emission. Upon changing the polarity, i.e. applying negative voltage to E1/E3, the device emits yellow light as it is now the yellow emitting sub-unit that is biased in forward direction. This polarity-dependent emission is shown in Figure 2.

\section{Materials and Methods}

In order to study the influence of the blue emitting sub-unit on the performance and color-tunability of our AC/DC OLEDs, we investigate blue sub-units based on three different blue fluorescent emitters: 4P-NPD, PPIP, and MADN:TBPe (Fig. 3; see full chemical names below.)

As p-type layer, we use 2,2',7,7'-Tetrakis-(N,N-di-methylphenylamino)-9,9'-spirobifluoren (Spiro-TTB) doped with 4 wt\% 2,2'(perfluoronaphthalene-2,6-diylidene)dimalononitrile (F6-TCNNQ). The n-type layers consist of 4,7-diphenyl-1,10-phenanthroline (BPhen) doped with cesium (Cs). For the blue emission layer (EML), either N,N'-di-1-naphthalenyl-N,N'-diphenyl-[1,1':4',1":4",1'"-quaterphenyl]4,4"'-diamine (4P-NPD), 4,4'-bis(1-phenyl-1H-phenanthro[9,10-d]phenanthroimidazol-2-yl)biphenyl (PPIP), or 2-Methyl-9,10bis(naphthalen-2-yl)anthracene (MADN) doped with $1.5 \mathrm{wt} \%$ of 2,5,8,11-Tetra-tert-butylperylene (TPBe) is used. The yellow EML is a double emission structure, combining the primarily hole-conducting 4,4',4"-tris(N-carbazolyl)-triphenylamine (TCTA) matrix and electronconducting 2,2',2"-(1,3,5-phenylen)tris(1-phenyl-1H-benzimidazol) (TPBi) matrix, both doped with 8 wt\% of the phosphorescent yellow 
emitter Bis(2-(9,9-dihexylfluorenyl)-1-pyridine)(acetylacetonate)iridium(III) (Ir(dhfpy)2(acac)). Electron and hole blocking layers (EBL/HBL) confine charge carriers and excitons to the EML. In case of the yellow emitting unit, aluminum(III) bis(2-methyl-8quninolinato)-4-phenylphenolate (BAlq2) and 2,2',7,7'-tetrakis-(N,N-diphenylamino)-9,9'-spirobifluorene (Spiro-TAD) are used as HBL and EBL, respectively. The HBL/EBL combination is different for the blue emitting units. In case of 4P-NPD and PPIP, we use BPhen/Spiro-TAD and BPhen/N,N'-Di(naphthalen-1-yl)-N,N'-diphenyl-benzidine (alpha-NPD), respectively. For MADN:TBPe, the HBL is BAlq2 whereas the EBL is alpha-NPD.

All layers are deposited in a UHV chamber at a base pressure of about $10^{-8}$ mbar onto glass substrates coated with structured tin-doped indium oxide (ITO) which acts as transparent bottom electrode. The highly transparent central multilayer electrode is a combination of 2 $\mathrm{nm}$ gold $(\mathrm{Au})$ and $9 \mathrm{~nm}$ silver $(\mathrm{Ag})$. A $100 \mathrm{~nm}$ thick layer of aluminum (Al) is used as highly reflective top electrode.

The current-voltage-luminance $(j-V-L)$ characteristics of our devices were measured with a source measure unit (SMU 2400, Keithley) in combination with a calibrated Si photo-diode. A calibrated spectrometer (CAS 140, Instrument Systems) recorded the spectral radiance in forward direction. Angular dependent measurements are used to determine the external quantum efficiency $(E Q E)$ and luminous efficacy $(L E)$ of the blue and yellow units, respectively, and were performed in a spectrogoniometer setup including a calibrated Ocean Optics USB4000 miniature spectrometer. Electrical characteristics under AC drive were measured using a high-precision digital power meter (WT1600, Yokogawa). The luminous flux under AC conditions was obtained in a calibrated integrating sphere (LMS-100, labsphere, $\mathrm{d}=$ $250 \mathrm{~mm})$.

\section{Ultrathin metal electrodes}

For the AC/DC OLED concept to work efficiently, the intermediate electrode (E2 in Fig. 1) has to provide a good combination of conductivity and optical transmission. At this position in the device the use of electrodes that are based on transparent conductive oxides, like ITO, is challenging as the sputtering or tempering procedures required to obtain high-quality ITO are likely to damage the underlying organic layers. Solution processable electrodes based on silver nanotubes [20] or PEDOT/PSS [21] are promising alternatives, but in the present scenario again challenging as the solvents used may attack the first OLED subunit. We therefore opted for the use of ultrathin metal films.

In our recent systematic study of ultrathin film metal films [19] we characterize the transmittance and sheet resistance of the different metal film configurations by depositing the electrodes on $20 \mathrm{~nm}$ thick hole-transport layers (HTL) on a glass substrate. This ensures that the growth conditions are similar to those in complete OLEDs while at the same time simplifying sample preparation and characterization. A $30 \mathrm{~nm}$ thick capping layer is deposited on top to mimic the situation within a device. A neat ITO electrode on the glass substrate is evaluated as a reference.

The ITO electrode shows a nearly constant transmittance between 81 and $88 \%$ across the entire visible part of the spectrum and has a sheet resistance of $32 \Omega / \square$. A $15 \mathrm{~nm}$ thick Ag layer, a thickness commonly used as semitransparent top electrode, shows a narrowed transmittance spectrum, decreasing from $76 \%$ peak transmittance at $425 \mathrm{~nm}$ to a minimum below $40 \%$ in the deep red. While the transmittance is thus significantly reduced compared to the ITO electrode, the sheet resistance also decreases to $3.9 \Omega / \square$. Intuitively, one would expect that decreasing the metal layer thickness below $15 \mathrm{~nm}$ will lead to an enhanced transmittance. However, silver and other metals tend to form isolated nuclei when deposited as a film with a thickness in the few nm-range [22]. For a nominal thickness below $7 \mathrm{~nm}$, silver layers grown directly on the HTL are non-conductive, i.e. show a sheet resistance $>10^{6} \Omega / \square$. In addition, the transmittance of $7 \mathrm{~nm}$ thick neat silver films is not larger than for the $15 \mathrm{~nm}$ thick film and the transmittance even reduces for yet thinner silver layers. This effect is attributed to plasmonic absorption of the isolated silver nuclei forming in the thinner films.

By applying a wetting layer system formed e.g. by a $\mathrm{MoO}_{3}$ - Au double layer underneath the silver film, the growth of the evaporated Ag improved and planar and homogeneous layers were obtained, even for total thicknesses of the metal layer below $10 \mathrm{~nm}$. The wetting layer electrode shows a transmittance similar to ITO, while exhibiting a lower sheet resistance of only $9.0 \Omega / \square$ compared to $32 \Omega / \square$ for ITO. Furthermore, the homogeneous wetting layer metal electrodes do not show parasitic plasmonic absorptions and therefore achieve a nearly constant transmittance of $70-80 \%$ across the range of the spectrum that is covered by the emitter molecules used in this study. While the $\mathrm{MoO}_{3}$ - Au double layer provided optimal performance on HTLs, we find that on ETLs like BPhen:Cs, Au on its own already provides optimal performance.

\section{Characterization of AC/DC OLEDs}

Figure 4 shows $j-V-L$ characteristics for each individual emission unit, as well as the $E Q E$ and $L E$ of each unit. For a negative bias, only the yellow unit is active and the choice of the blue emitter should not influence the performance. The $j$ - $V$ characteristics as well as the $V$ - $L$ curves of the yellow unit are indeed identical for samples using different blue fluorescent emitters (Figure 4a). As expected, the efficiency differences between the individual samples are also within the sample-to-sample variation (Figure $4 \mathrm{c}$ ). At a brightness of $1,000 \mathrm{~cd} \mathrm{~m}^{-2}$, we obtain EQEs of $15-16 \%$ and $L E$ s of $50-55 \mathrm{~lm} \mathrm{~W}^{-1}$ for the yellow units in the different samples. For the three different blue emitters, we find a very similar $j-V$ behavior and observe only small differences in the $V$ - $L$ characteristics (Figure $4 \mathrm{~b}$ ). In terms of efficiency, the AC/DC OLED with 4P-NPD - a deep-blue emitter - shows the lowest $L E$ of around $1.2 \mathrm{~lm} \mathrm{~W}^{-1}$ at $1,000 \mathrm{~cd} \mathrm{~m}^{-2}$ whereas the sky-blue emitter PPIP and the host:guest system MADN:TBPe provide $L E$ s of $2.3 \mathrm{~lm} \mathrm{~W} \mathrm{~m}^{-1}$ and $4.4 \mathrm{~lm} \mathrm{~W}^{-1}$, respectively, as shown in Figure $4 \mathrm{~d}$. The $E Q E$ of the three different blue units ranges from $2.5 \%$ in case of PPIP over $3.1 \%$ in case of 4P-NPD to around 3.4\% in case of MADN:TBPe. 
Upon application of an AC signal, the device alternately emits pulses of blue and yellow light. When using a sufficiently high frequency, the human eye cannot resolve the subsequent emission from each unit, but instead perceives a color equivalent to the integrated emission over several operation cycles. By modifying the ratio of pulse-width and/or pulse-height between positive and negative half-cycle of the AC signal, the relative contribution of blue and yellow emission can be adjusted, which allows to tune the emission color perceived by the human eye.

We also measure the luminous efficacy of AC/DC OLEDs based on each of the three tested blue emitters, with the devices tuned to warmwhite color coordinates using a $50 \mathrm{~Hz}$ alternation frequency between the blue and the yellow subunit. Color-tuning was performed by adjusting the "on-time" of each unit, i.e. by a variation of the relative pulse-widths of the positive and the negative half-cycle at equal and constant pulse-height/driving voltage.

Interestingly, when tuned to warm white and operated at $1,000 \mathrm{~cd} \mathrm{~m}^{-2}$, the AC/DC OLED with 4P-NPD reaches the highest luminous efficacy even though the luminous efficacy of the blue subunit with 4P-NPD alone was considerably lower than the efficacy of blue subunits with either of the other two blue emitters. For white emission the 4P-NPD devices reaches $36.81 \mathrm{~m} \mathrm{~W}^{-1}$, the PPIP device achieves $31.5 \mathrm{~lm} \mathrm{~W} \mathrm{~m}^{-1}$ and the MADN:TBPe devices achieves $27.91 \mathrm{~m} \mathrm{~W}^{-1}$.

This observation is explained by the difference in luminous efficacy between the two sub-units, with the phosphorescent yellow unit having a 40-fold higher luminous efficacy than the fluorescent blue unit. It is therefore important to maximize the on-time of the yellow emitter and keep the contribution of the blue emitter at a minimum. We find that this is best achieved with the deep-blue emitter 4P-NPD where the on-time of the blue unit for warm-white emission is merely $15 \%$. The more sky-blue emitters PPIP and MADN:TBPe require increased ontimes of $20 \%$ and $30 \%$, respectively, resulting in reduced luminous efficacy compared to the 4P-NPD sample.

\section{Conclusions}

We have demonstrated highly efficient color-tunable AC/DC OLEDs based on a combination of a blue and a yellow emitting unit [15] and ultrathin metal electrodes [19]. Fabrication and electrical driving of these devices has been simplified by reducing the number of independent electrodes to two and using an $\mathrm{AC}$ voltage for color tuning. In future work, this concept might be extended to three pinOLEDs stacked on top of each other, providing red, green, and blue emission. This configuration is particularly interesting for display applications where a vertical stacking of the red, green, and blue sub-pixel allows higher pixel densities and an optimal fill factor in comparison to conventional side-by-side fabrication.

\section{Acknowledgements}

This work was funded by the European Social Fund and the Free State of Saxony through the OrthoPhoto project. M.C.G. acknowledges support from European Union Marie Curie Career Integration Grant (PCIG12-GA-2012-334407).

\section{References}

[1] H. Sasabe and J. Kido. Development of high performance OLEDs for general lighting. Journal of Materials Chemistry C, 1:16991707, 2013.

[2] S. Reineke, F. Lindner, G. Schwartz, N. Seidler, K. Walzer, B. Lüssem, and K. Leo. White organic light-emitting diodes with fluorescent tube efficiency. Nature, 459:234-238, 2009.

[3] H. Sasabe, J. Takamatsu, T. Motoyama, S. Watanabe, G. Wagenblast, N. Langer, O. Molt, E. Fuchs, C. Lennartz, and J. Kido. HighEfficiency Blue and White Organic Light-Emitting Devices Incorporating a Blue Iridium Carbene Complex. Advanced Materials, 22:5003-5007, 2010.

[4] T. Komoda, K. Yamae, V. Kittichungchit, H. Tsuji, and N. Ide. Extremely High Performance White OLEDs for Lighting. SID 2012 DIGEST, pages 610-613, 2012.

[5] Y. Z. Wang, D. D. Gebler, L. B. Lin, J. W. Blatchford, S. W. Jessen, H. L. Wang, and A. J. Epstein. Alternating-current light-emitting devices based on conjugated polymers. Applied Physics Letters, 68:894, 1996.

[6] T. Tsutsui, S.-B. Lee, and K. Fujita. Charge recombination electroluminescence in organic thin-film devices without charge injection from external electrodes. Applied Physics Letters, 85:2382, 2004.

[7] J. Sung, Y. S. Choi, S. J. Kang, S. H. Cho, T.-W. Lee, and C. Park. AC Field-Induced Polymer Electroluminescence with Single Wall Carbon Nanotubes. Nano Letters, 11:966-972, 2011.

[8] M. Fröbel, S. Hofmann, K. Leo, and M. C. Gather. Optimizing the internal electric field distribution of alternating current driven organic light-emitting devices for a reduced operating voltage. Applied Physics Letters 104: 071105, 2014.

[9] M. Fröbel, A. Perumal, T. Schwab, C. Fuchs, K. Leo, and M. C. Gather. White light emission from alternating current organic lightemitting devices using high frequency color-mixing. Phys. Status Solidi A, 210:2439-2444, 2013.

[10] M. Fröbel, A. Perumal, T. Schwab, M. C. Gather, B. Lüssem, and K. Leo. Enhancing the efficiency of alternating current driven organic light-emitting devices by optimizing the operation frequency. Organic Electronics, 14:809-813, 2013. 
[11] M. Fröbel, A. Perumal, K. Leo, and M. C. Gather. White light emission from alternating current organic light-emitting devices using high frequency color-mixing. Proc. SPIE Optics and Photonics 8829-69, San Diego, CA, USA, 2013

[12] A. Perumal, M. Fröbel, S. Gorantla, T. Gemming, B. Lüssem, J. Eckert, and K. Leo. Novel Approach for Alternating Current (AC)Driven Organic Light-Emitting Devices. Advanced Functional Materials, 22:210-217, 2012.

[13] A. Perumal, B. Lüssem, and K. Leo. Ultra-bright alternating current organic electroluminescence. Organic Electronics, 13:1589-1593, 2012.

[14] A. Perumal, B. Lüssem, and K. Leo. High brightness alternating current electroluminescence with organic light emitting material. Applied Physics Letters, 100:103307, 2012.

[15] M. Fröbel, T. Schwab, M. Kliem, S. Hofmann, K. Leo, and M. C. Gather. Get it White: Color-tunable AC/DC OLEDs. Light: Science \& Applications 4:e247, 2015.

[16] Z. Shen, P. E. Burrows, V. Bulović, S. R. Forrest, and M. E. Thompson. Three-Color, Tunable, Organic Light-Emitting Devices. Science 276: 2009-2011, 1997.

[17] S. Lenk, T. Schwab, S. Schubert, L. Müller-Meskamp, K. Leo, M. C. Gather, S. Reineke. White organic light emitting diodes with 4 nm metal electrode. Applied Physics Letters 107:163302, 2015.

[18] T. Schwab, S. Schubert, L. Müller-Meskamp, K. Leo, M. C. Gather. Eliminating Micro-Cavity Effects in White Top-Emitting OLEDs by Ultra-Thin Metallic Top Electrodes. Advanced Optical Materials 1:921-925, 2013.

[19] T. Schwab, S. Schubert, S. Hofmann, M. Fröbel, M. Thomschke, L. Müller-Meskamp, K. Leo, M. C. Gather. Highly Efficient Color Stable Inverted White Top-Emitting OLEDs with Ultra-Thin Wetting Layer Top Electrodes. Advanced Optical Materials 1:707-713, 2013.

[20] W. Gaynor, S. Hofmann, M. G. Christoforo, C. Sachse, A. Salleo, M. D. McGehee, M. C. Gather, B. Lüssem, L. Müller-Meskamp, P. Peumans, K. Leo. Color in the Corners: ITO-Free White OLEDs with Angular Color Stability. Advanced Materials 25:4006-4013, 2013.

[21] Y. H. Kim, J. Lee, S. Hofmann, M. C. Gather, L. Müller-Meskamp, K. Leo. Achieving high efficiency and improved stability in ITOfree transparent organic light-emitting diodes with conductive polymer electrodes. Advanced Functional Material 23:3763-3769, 2013.

[22] K. Leosson, A. S. Ingason, B. Agnarsson, A. Kossoy, S. Olafsson, M. C. Gather. Ultra-thin gold films on transparent polymers. Nanophotonics 2:3-11, 2013. 


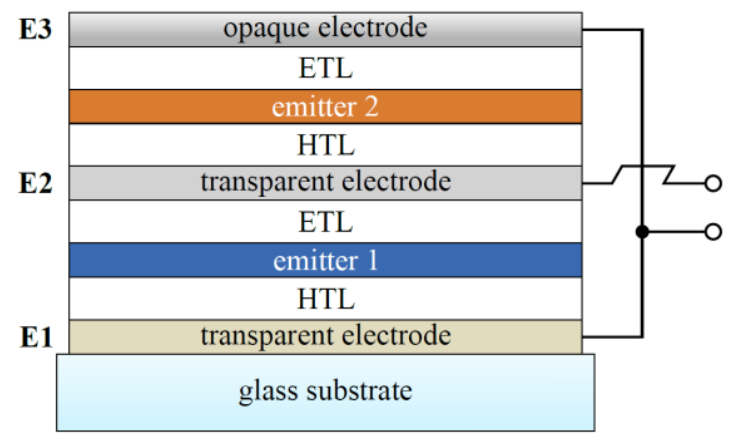

Figure 1. Schematic illustration of the architecture of an AC/DC OLED with electrodes and wiring. Electrodes E1 and E3 are connected to each other and E2 is the independent counter electrode, allowing for two-terminal device operation using only one power supply. 

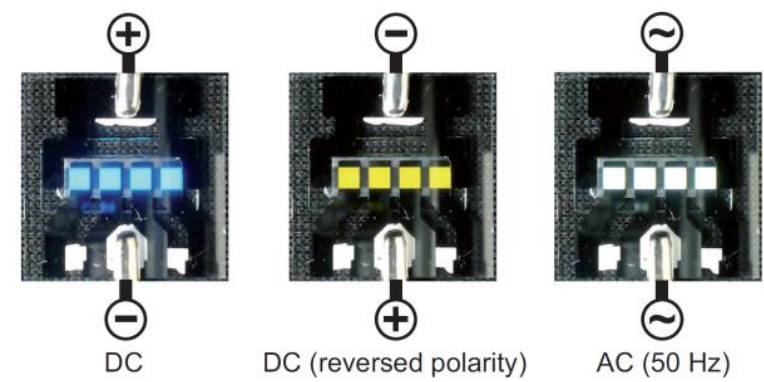

Figure 2. Pictures showing an AC/DC OLED sample upon application of a DC bias (blue emission), a DC bias with reversed polarity (yellow emission) and a $50 \mathrm{~Hz}$ AC bias (white emission). 


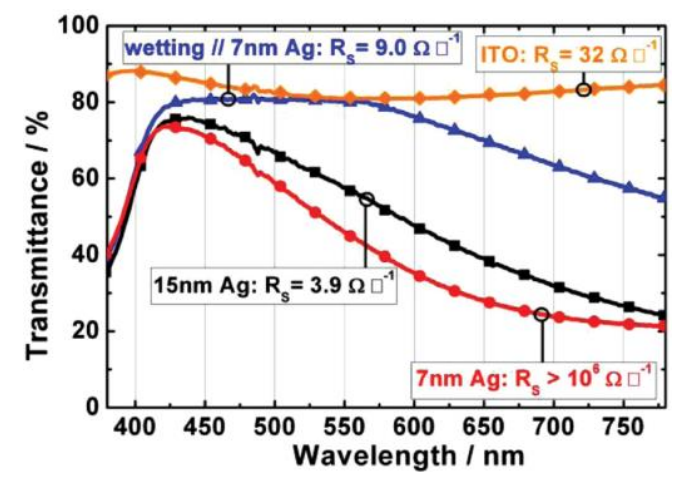

Figure 3. Transmittance spectrum and sheet resistance of different thin metal films and of commercial ITO. 


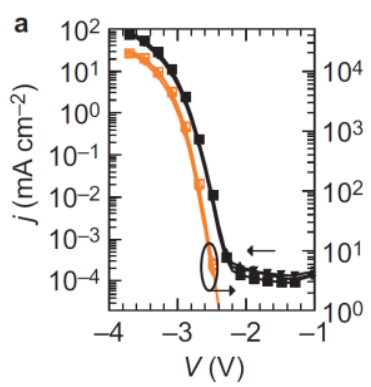

b
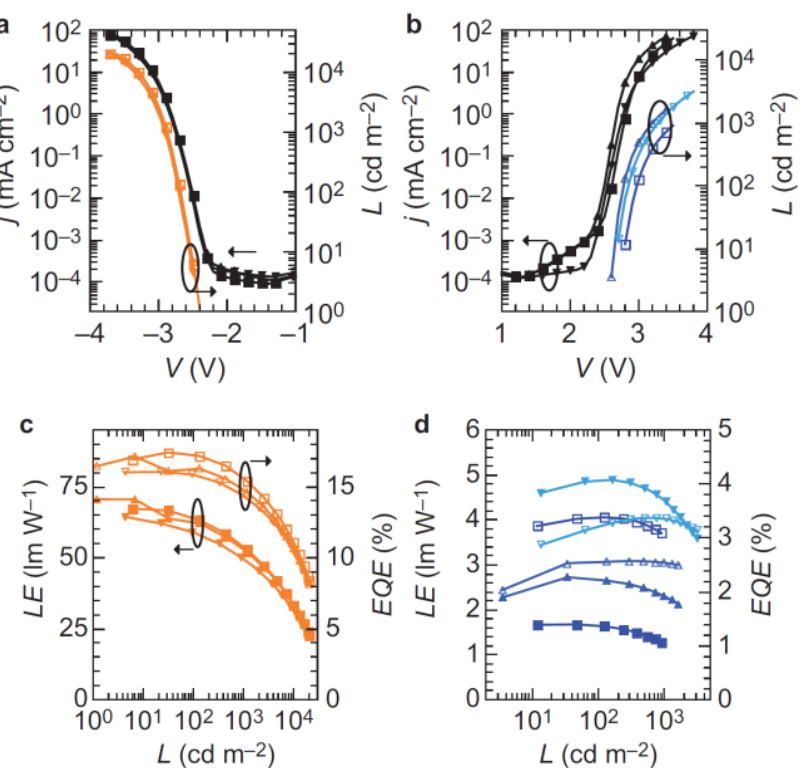

Figure 4. Current density (j) and luminance (L) versus voltage (V) for (a) the yellow-emitting units and (b) the blue-emitting units of devices based on three different blue emitters, 4P-NPD (squares), PPIP (up-facing triangles), and MADN:TBPe (down-facing triangles). (c) and (d) show the corresponding luminous efficacy (LE, closed symbols) and EQE (open symbols) versus device luminance for the yellow and blue units, respectively. Data were acquired during DC operation. 